pressure upon it from behind increases, breaks from its attach. ment at its base, is carried upwards by the force of the blood-current into the narrower part of the vessel, and, completely obstructing the life-stream, leads to inevitable and almost immediate death.

[To be continued.]

\section{obringual Communications.}

\section{THE PATHOLOGY, DIAGNOSIS, AND TREATMENT OF CARDIAC DISEASES.}

By W. O. Markham, M.D., F.R.C.P., Physician to St. Mary's Hospital, London.

VIII.-Endocarditis : Pathology. Acute Affections or the Heart's Valves: their Degenerations.

By the term endocarditis is understood inflammation of the whole or of a part of the membrane which covers the valves and the internal surface of the heart. Included also under this head are all those morbid changes which the valvular structures undergo in connexion with acute diseases, or which are indirectly the consequences of the endocardial inflam. mation.

Much obscurity still involves the pathological history of endocarditis; for now-a-days, happily, we have very rarely an opportunity of studying the disease in its early stage. The remarkable researches and experiments of Dr. Richardson appear, however, to throw some light upon this subject ; and I shall, therefore, state shortly what he has observed in cases of endocarditis induced artificially by the injection of lactic acid into the peritoneum.

In considering this subject, it is necessary to keep in view the anatomical nature of the endocardial membrane, and the peculiar accidental circumstances which attend upon and influence its inflammation. This membrane is continuous with, and very similar in structure to, that which lines the arteries and veins. It consists of a stratum of fine fibres, bearing upon them a layer of very delicate epithelium. Blood-vessels ramify in the fibrous tissue, and it is from these that the pathological products of the inflammation are derived.*

The peculiar circumstances attending this inflammation are, the unceasing motion of the part inflamed; and the mechanical action of the blood, which is constantly flowing over and is in contact with the inflamed surface, and with any exudations which may escape from it.

The deep red colour, which the internal surface of the hesrt frequently presents after death, was once considered as an in variable sign of inflammation of the endocardium. It is, however, in almost all cases, the result simply of imbibition by the heart's tissues of the decomposed and dissolved colouring matter of the blood.

The redness of congestion - the true inflammatory rednessis described by Rokitansky as constantly presenting a pale rose-red colour, the tint of it being subdued by the superficial layers of the endocardial membrane; it assumes a stripe-like, ramifying appearance, corresponding to that of the vessels, and has not the appearance of a general saturation of the tissues. Small ecchymoses are seen here and there in the inflamed part.

In the first stage of the inflammation, Dr. Richardson (On the Coagulation of the Blood, p. 371, et seq.) found the endocardial surface "intensely vascular, approaching in colour to bright vermilion; the membrane also had a soft fleecy character, giving to it a velvety appearance". The valves were at the same time swollen and vascular, and clear lymph oozed from them when pricked. This lymph appears occasionally to have transuded through the membrane, and may have thus laid the foundation for fibrinous deposits from the blood, by causing its coagulation-an important observation, if further corroborated. "On the surface of the valve (mitral) there was a small fibrinous deposit, which adhered to its position by

* The idea, that the internal membrane of the heart or arteries is nou rished by the blood which flows over it, is quite contrary to all physiological
facts. means of an underlayer of a white glutinous fluid, the like of which I found afterwards could be made to exude from the valve on puncturing it with a needle." Small beads were at the same time developed in the margin of the valves, and also yielded a clear coagulable lymph when pricked, and then shrunk away.

In the second stage of the inflammation, the endocardial surface became less vascular, and had a pale pink colour. The valves also were less red, but were still thickened with the now semisolid exudation within them; and at points their tendinous chords were attached by loose adhesions to the endocardium. ln the third stage, all redness disappeared; the exudation was firmer, and the valves and their beaded edges for a long time retained considerable firmness, and a pearly whiteness.

From these observations, it would appear, that the ordinary products of inflammation are exuded both upon and within the endocardium. There are, therefore, two kinds of endocardial deposits to be considered : The "beads," the granulations, and thickenings, which are produced solely by the inflammatory exudation thrown out beneath the unbroken endocardial surface, and therefore within the valves; and the "vegetations," etc., formed chiefly from the fibrine of the blond flowing through the heart, which is attracted to and deposited either upon the surface of the inflamed membrane, or upon the inflammatory matter that has exuded through it. These vegetations, therefore, are engrafted on the inflammatory deposit. It seems very probable, indeed, that a slight degree of roughness of the endocardial surface, resulting from the inflammatory action, may thus induce a deposition upon it of the fibrine of the blood; so that in this way the inflammation becomes, indirectly, a cause of the formation of fibrinous coagula. And this is still more probable in diseases, such as acute rheumatism, in which the blood appears to bave a special tendency of separating from its fibrinous constituents.*

These abnormal products, thus formed upon or exuded within the inflamed endocardium, present themselves to us under a variety of forms, differing much in size, number, and consistence. At one time, they exist as minute granulations clustered together around the bases, or fringing the borders of the valves; sometimes they are attached, like polypoid bodies, to the valves, in an isolated form, varying in size from a pea to a hazel-nut. Then, again, they take the form of cauliflower excrescences, passing between and separating the valves, or fringing their torn and roughened edges. The fibrinous deposits are also frequently found fixed, not only on the valves, but also on the endocardium in their neighbourhood. They are mostly found fixed to that surface of the valves which is turned towards the current of the blood flowing through the heart. The different appearances which these granulations and vegetations, etc., present are readily explained, when we regard them as being formed, first, by the deposition of fibrine of the blood upon an inflamed surface; and, secondly, as the results of inflammatory exudation, having the endocardial membrane still unbroken upon them. Whenever this surface is broken or roughened by inflammation, a deposition of the fibrine of the blood (as it would appear) readily takes place upon the broken part.

The valves, and their chords, are not unfrequently ulcerated and ruptured, as a consequence of the endocardial inflammation. The tendons of the papillary muscles, and the valves themselves, are also sometimes fused together, or with the neighbouring parts, and so as to render the closure of the valves imperfect, or to diminish the aperture they surround. The aperture is also sometimes constricted by the deposit of inflammatory exndation in the tissues which form its circum ference.

Endocarditis occurs more frequently, or at least its action is much more severe, in the left than it is in the right side of the heart. + The resolution of it is rarely perfect. Whenever we are able to diagnose the presence of endocarditis, we generally find that some appreciable consequences of the inflammation remain after the inflammatory symptoms have passed away.

* Virchow asserts, that these fibrinous formations are merely layers of fibrine separated from the current of blood which flows over the mernbran The only difference which exists, in his view, between endocarditis and endarterits, is in the more acute course of the former, and in the tendency of it to the formation of papillary or warty excrescences. Both these facts he attributes to the greater vascularity and looseness of the cellular tissue which lies between the valves and beneath the inner layer of the endocardium.

+ I am inclined to think that endocarditis attacks the right side of the heart more frequently than is generally supposed; and that we do not notice the fact, because its effects are much less severe, and the pathological signs of it less well marked. In most cases, when the mitral valve is much diseased, the tricuspid will, I believe, be found also affected. 
Under very favourable circumstances, it is possible that the in. flammatory products may be entirely removed. The fluid exudation may be absorbed, and the fibrinous portions of them - the vegetations-formed out of coagula of the blood, be removed by the disintegrating action of the blood which flows over them. The fibrinous particles thus removed from their points of attachment, and carried along in the current of the blood into the general circulation, occasionally give rise to secondary disorders in the lungs, the brain, the kidneys, and other organs of the body, and sometimes appear to infect and alter the constitution of the blood itself.

The formation of abscess in the walls of the heart, as a result of endocarditis, is a most rare occurrence; when it exists, it may occasion rupture of the heart's walls.

Endocarditis is especially of interest, as being a cause of valvular diseases. Its chief effects, indeed, appear to fall upon the valvular apparatus; and when this has not been injured by the inflammation, and when the muscular tissue of the heart has not been damaged by it, the consequences of endocarditis are of minor importance.*

Chronic Valvular Diseases. Diseases of the valves of the heart, however, are not the products solely of inflammation. They are also, and perhaps in the majority of cases which occur at advanced periods of life, the consequences of a slow degeneration of the textures which form the valves. Chronic diseases of the valves are obscure in their origin, and slow and insidious in their progress. It is only in their advanced states, when they have so destroyed the integrity of the valve's structures as to occasion them imperfectly to perform their office, that they make their existence known to us during life by physical signs and symptoms. They present themselves to us under the form of abnormal depositions of fibrinous, atheromatous, and calcareous matters, beneath the endocar dium, and within the valves. The quantity, and form, and nature, and stage of the deposit in each particular case, is the measure of the amount of injury done to the valve which it has pervaded. These peculiar degenerations of the textures of the heart and vascular system generally are, in one shape or other, so frequently observed in advariced life as to lead to the ides that they are modes of the natural decay of the tissue. When met with at early periods of life, they must be regarded as the consequences of perverted nutrition, resulting from un healthy conditions of the system. There is reason to believe, for instance, that the fibrinous and atheromatous deposits in the valves and arteries are promoted by excessive indulgence in alcohelic fluids; and there can be no doubt that all habits and causes which tend to weaken and injure the system generally, assist in promoting any existing tendency to the deposit of these materials in the tissues. It is also well to remember, that the true inflammatory exudations may also undergo these degenerative changes.t

Valvular diseases, as here considered, result from the destruc tion and disintegration of their tissues when invaded by these abnormal-fibroid, fatty, and calcareous-matters. The natural properties of these tissues-their remarkable strength, firm ness, and elasticity - are diminished or destroyed when invaded by these matters; so that the tissues become softened and brittle, and unfitted for their function, the consequence of which is that they tear, rupture, and yield under the pressure and distension to which they are of necessity continually sub jected by the action of the heart. 'The deposits take place, for the most part, beneath and within the internal membrane of the valves of the heart and vessels, and consequently they cut off its nutritive supply, which, in the case of the arteries, is derived from the vasa vasorum, through the medium of the middle coat. Hence, also, arises a cause of ulcerations and destructions of parts. The deposit is at one time chiefly atheromatous, and soft; at another, by the accumulation of calcareous salts, the tissues become hardened, brittle plates of greater or less extent being thus formed in it, and sometimes so extensively as to convert the valve or the artery affected into a brittle, hardened mass. Under such circumstances, when the internal membrane is broken or destroyed, the roughened borders and points of the calcareous plates become exposed to immediate contact with the blood, so that the fibrine of this fluid not unfrequently becomes attracted to and deposited upon

* It must not be forgotten, liowever, that the inflammation may be and frequently is, the cause of a slow degeneration of the muscular tissue, noted than the valvular injury, because it appears less immediately con. nected with the inflammation.

+ Under this head, I would particularly call attention to some very inter. esting researches of $\mathrm{Mr}$. Canton, which certainly tend to some very interatheromatous degeneration being a special attribute of old age.

$$
126
$$

them, just as we found it was in the case of endocarditis. The blood, also, under such conditions, is apt to assist in the further destruction of the affected parts, being forced at each systole of the heart beneath the edges of the calcareous plates, and against the softened and weakened tissue. It is in this way that it, usually, gradually distends the arterial coats in the formation of aneurisms, and aids in the rupture and ulceration of the valves of the heart.

We not unfrequently meet in practice with cases in which one of the aortic valves appears to have been suddenly ruptured in this way by violent muscular exertion. The individual affected may have enjoyed perfect health up to the date of such exertion; but from that moment has suffered from symptoms of heart affection. We may reasonably conjecture that, in such cases, the tissues of the valves must have been partially degenerated, and though equal to ordinary pressure, were unable to bear that which resulted from the extra exertion. It is hardly possible to conceive that any degree of inordinate action of the circulating organs could occasion rupture of perfectly healthy valves. The two following cases will exemplify the point here spoken of. I have notes of several others :-

A. B., a gardener, about 54 years old, had always enjoyed excellent health until about twelve weeks ago. He dates his illness from a "strain" which happened to hin when wheel ing a heavy wheelbarrow. From that moment he has never " been himself," and his breathing has been affected. He was, at the time he came to me, suffering from decided symptoms of heart-disease; and auscultation pointed out defect of the aortic valves, there being a loud diastolic murmur audible over the site of the aortic valves, and down along the sternum. There can be no rational doubt, that in this case the aortic valvular injury occurred at the time of " the strain."

In another case, the subject of the aortic valvular disease was a man about 45 . He had always been very active, and was an excellent runner, and, in fact, used to boast of his wind. About eight months before his death, while running quickly on message, he was suddenly seized with violent pain in his left side, and in consequence with difficulty reached his home again. From this moment his breathing became affected. I saw him about five months afterwards, and found marked signs of hyper. trophy and aortic valvular disease. He eventually sank, after suffering many most severe angina-like attacks. In this case, after death, I found the aortic valves all incompetent; two of them were partially destroyed by ulceration; two of them, also, were puckered, and fused together at their adjoining angles. The aorta, the lining membranes, and the other valves of the heart, were perfectly healthy. 'The heart was greatly hypertrophied. There was no other disease of parts. Is it not fair to conclude, that in this case the heart-disease dated from the time of the great exertion made in running? His breathing had been previously good, and afterwards always bad. There was no other cause to account for the disease; no rheumatism or kidney affection, etc.

Valvular diseases we therefore find have different modes of origin. They may be the consequences of inflammatory action, and they may be the consequences of textural degenerations. Inflammation softens the valves, in part destroys their tissues, and so renders them liable to ruptures and bulgings. Its exudation into them gives rise to fibrinous formations, whereby they are thickened, and rendered less pliable, firm, and elastic than natural. Hence, also, arise contractions of the valves, their tendons, and their orifices, and adhesions of them to each other, and to the cardiac walls. The fibrinous vegetations (polypoid growths) which form upon their edges, surfaces, and around their tendons, also project between the valves, and prevent their perfect closure. Atheromatous and calcareous degenerations of the valves cause them to assume most varied forms. Sometimes mere specks of the abnormal deposit are seen scattered in the tissues, and sometimes the valves and the arteries are through it converted into semisolid structures. The calcareous matter, again, may perforate the valves, and assume the most fantastic shapes; thereby, and by deposit of it in the surrounding tissues, the aortic orifice, for instance, may be rendered scarcely large enough to admit the passage of a quill; and so likewise, and by ad. hesions, the mitral valves may be reduced to the condition of a solid cone, projecting into the left ventricle, having an opening through it scarcely large enough to admit the point of the little-finger. The destructive tendency of these degenerations, and the degree of obstruction to the circulation, and of re gurgitation of the blood, which they occasion, our pathological museums abundantly illustrate. Defects of the valves, I may 
add, may also result from original malformations of them. Thus, for instance, there may be one valve only at the aortic orifice, or two of the valves may adhere together, or the three may be present, and one of them very small and in a rudimen. tary state. Congenital defects of the valves of the pulmonary artery are generally associated with other serious organic defects of the heart or its vessels, such as are incompatible with prolonged life. They are therefore rarely met with, except in the very young.

\section{REMARKS ON DR. SKINNER'S CASE OF SUSPECTED POISONING BY OXALIC ACID.}

By J. L. W. Thudichum, M.D.

The last number of the Britrsh Medical Journal contains an account of a case of suspected poisoning by oxalic acid or its salts, by Dr. Skinner of Liverpool, upon which $I$ beg to offer a few remarks.

An unmarried female was on the Sth of November seized with a severe rigor, followed by an attack of acute peritonitis. Twelve leeches were applied to the abdomen; and the ordinary ice and opium treatment was adopted. Seven days after, the patient was convalescent. On November 20 th, a relapse occurred. T'he peritonitis, which before was general, was now limited to a small spot in the left umbilical region. There was occasional vomiting and retching, etc.; consequently the tongue was furred, and the thirst intense. Eight leeches were applied over the painful spot, followed by large anodyne poultices, and ice and opium. In two days, the patient was again convalescent. On December 1st, the patient complained of continued nausea, drank warm water, and accordingly vomited; but the nausea continued. On December 2 nd, she had been vomiting more or less during a space of twenty-four hours, and was apparently rapidly passing into collapse. Creasote and morphia were given; mustard poultices were placed on the hypogastrium ; and the patient surrounded with bottles of hot water. Later, hydrocyanic acid was given in effervescence, with bicarbonate of potash and lemon-juice. Beef-tea and port wine were administered per anum. In the evening, vomiting had entirely ceased, and reaction was fairly established. Next day she had "twitchings", during the occurl'ence of which a copious flow of dark coloured fluid passed from the patient per vaginam. So copious was it, that it saturated the napkin and the bedclothes. In a day or two from the date of the excessive vomiting, the patient was thoroughly convalescent. Six ounces of the fluid vomited by the patient on December 2nd were examined, and found to contain no poison. The patient, who is described as healthy, had on a previous occasion six leeches applied to her, on account of inflammation of the cervix uteri.

This is the essence of the case, divested of its atmosphere of suspicion.

Giving Dr. Skinner full credit for the correctness of his diagnosis, so far as regards the existence of peritonitis, this would probably be the first case on record in which the cause of such a clear acute attack of peritonitis is ever alleged to have been an irritant poison. But, even admitting that such symp. toms as those observed during the second and third attacks might be produced by oxalic acid, there remains one symptom which cannot be explained by the assumption of poisoning simply ; namely, the bloody discharge at the crisis of the dis. order, two days subsequently to which the patient was thoroughly convalescent. Dr. Skinner is not able to account for this discharge of blood, which produced stains resisting all domestic efforts at removal.

This blood was evidently not ordinary blood, coming direct from an artery or vein, or from capillary vessels; for such blood does not produce stains resisting the domestic efforts at removal. For the same reason, it cannot be considered as fresh menstrual blood, particularly if we give credit to the statement that the catamenia had appeared shortly before her illness. But if we consider this blood to have been effused into some internal cavity some time before its discharge, and there to have been retained and altered, then the colour of the fluid, the copiousness of its flow, and the peculiar nature of the stains, are satisfactorily accounted for.

Now, as peritonitis preceded by rigors, being itself a very rare disorder, is not produced by oxalic acid taken into the stomach, and as a sudden discharge of blood from the vagina, or perhaps the discharge of an accumulation of blood from the uterine cavity, or the accumulation of blood in the uterine cavity, are not symptoms or sequels of oxalic acid introduced into the system, we have here the two most important elements in the disorder, the beginning and the critical end, which absolutely deny the possibility of poisoning by oxalic acid.

On the contrary, if we assume the large quantity of peculiar blood to have existed in the uterine cavity at the beginning of the disorder, on November 8th (which, in a female who had before been suffering from inflammation of the cervix uteri, so that six leeches had to be applied to the os uteri by Dr. Skinner himself, is an assumption supported by great probability), then all the symptoms of peritonitis, general and local, the romiting, the nauser, the twitches, and the discharge, the amenability of the case to treatment, the very rapid and complete recovery, are satisfactorily accounted for.

No poison was found in six ounces of matter which had been vomited by the patient. During the night preceding this particular act of vomiting, oatmeal gruel had been administered every half hour or so, but always came up instantaneously. Now this very oatmeal gruel, being the suspected vehicle, should reasonably have had a peculiar acid taste, had it contained oxalic acid-a taste which the patient must have noticed, and which she could not merely describe as a taite different from that of tea and other liquids of which she partook about the same time. The taste of oatmeal gruel is distinctly different from the taste of tea and other liquids, even when that gruel is quite pure and unadulterated with oxalic acid. The avidity. with which the patient says she swallowed the gruel, is an afterthought, and evidently invented to account for the real absence of any peculiar taste of the gruel. Some of this gruel, given every half hour, must have been in the stomach at the time the vomiting occurred, the produce of which was analysed, and found to contain partially digested starch-granules, but no oxalic acid. It was an afterthought of the patient, of which she informed Dr. Skinner, that she had similar gruel before this illness, which never did more than nauseate, and that she suffered from " everlasting nausea", "now and again", for the last two years. The patient evidently neither remarked nor said anything about her gruel, either during the last two years or this present illness: it was all elicited from her by the sug. gestion of poisoning, and then nothing was elicited supporting in any way that suggestion.

The following statement is adduced in support of the case having been one of poisoning by oxalic acid:- "I have been told since, that they" (the twelve and eight leeches applied on two occasions) " did not fix well, that they soon came off, and, with one exception, they all died immediately, the only exception dying next day." But the leeches which the author himself had formerly applied to the os uteri had all lived long after that service. A case by Dr. Arrowsmith, reported by Christison, has evidently suggested the inquiry about the leeches.

Had the information about the leeches been obtained immediately after the event, it could perhaps have been credited. But, regarding this piece of information, all control is wanting. It is not even said who informed Dr. Skinner of the dying of the leeches; it is not said who administered the leeches, who took them off, and how they were treated. A leech kept in the dry over night, in a warm room, particularly if it has been roughly handled, is almost sure to die. Leeches frequently fix badly, or soon come off, even when they have been sucking quite healthy blood. Leeches also have been observed to die after use upon patients who certainly did not suffer from poisoning by irritant poison. Further, in the case of Arrowsmith, the proof is yet wanting that those leeches which died, died in consequence of the admixture of oxalic acid with the blood, or in consequence of any change in the blood produced by oxalic acid. This whole statement about the leeches is, in our present case, a baseless fabric of vision.

In a medical point of view, then, the case offers no peculiarity whatsoever. It was a case of chronic disease of the uterus, either chronic inflammation of the cervix, or its consequences, narrowing or obliteration of the cervical canal; or, what is also very likely, and accounts better for the alleged regularity of the catamenia, it may have been a case of antero- or retroflexion of the uterus, diseases which are not rarely accompanied by inflammation of the cervix uteri. As is very common in such cases, catamenial blood was retained in the cavity of the uterus, and altered in the usual manner. This condition, then, produced the very common symptoms connected with the nervous system, recurring or intermittent attacks of pain in the abdomen, which pain was treated as peritonitis, but is perhaps more properly described as uterine colic, with irradiation of the hyperæsthesia over some or all of the 